\title{
Probing Bright and Dark Surface-Plasmon Modes in Individual and Coupled Noble Metal Nanoparticles Using an Electron Beam
}

\author{
Ming-Wen Chu, ${ }^{,, \dagger}$ Viktor Myroshnychenko, ${ }^{, \neq}$Cheng Hsuan Chen, ${ }^{+, \S}$ \\ Jing-Pei Deng, ${ }^{\prime \prime}$ Chung-Yuan Mou, ${ }^{\perp}$ and F. Javier García de Abajo ${ }^{\ddagger}$
}

Center for Condensed Matter Sciences, National Taiwan University, Taipei 106,

Taiwan, Instituto de Óptica-CSIC and Unidad Asociada CSIC-Universidade de Vigo,

Serrano 121, 28006 Madrid, Spain, Department of Physics, National Taiwan

University, and Institute of Atomic and Molecular Sciences, Academia Sinica,

Taipei 106, Taiwan, Department of Chemistry, Tamkang University,

Taipei County 25137, Taiwan, and Department of Chemistry, National Taiwan

University, Taipei 106, Taiwan

Received October 29, 2008; Revised Manuscript Received November 25, 2008

\begin{abstract}
The rich structure of bright and dark surface-plasmon modes localized in individual and coupled gold nanoparticles is unveiled by electronenergy-loss spectroscopy performed in a scanning transmission electron microscope. Spatially resolved maps of surface-plasmon modes in the $\sim 1.5-2.5 \mathrm{eV}$ range (wavelengths $\sim 500-800 \mathrm{~nm}$ ), collected for individual nanorods, coupled nanorod dimers, and touching nanosphere dimers, are in excellent agreement with theory. Surface-plasmon maps constructed from the spatially and spectrally resolved energy-loss signals are shown to mimic rather well the near fields calculated for external illumination in the case of bright surface-plasmon modes (i.e., those coupling to external light). Dark surface-plasmon modes that cannot be excited by optical means are also found, and our electron probing technique provides further insight into their corresponding spatial distribution and symmetry, which are not accessible to any other existing techniques. Our results initiate the study of a whole set of new dark surface-plasmon modes that should become a source of new applications in sensing and microscopy but have escaped experimental scrutiny so far.
\end{abstract}

Surface plasmons (SPs) are quantized collective oscillations of conduction electrons propagating at the surface of metals. ${ }^{1-3}$ These excitations lie in the visible and near-infrared regime (vis-NIR) for noble metals, thus dictating their color by coupling to light. ${ }^{4}$ Optically excited SPs are featured by dipolar charge oscillations ${ }^{5-7}$ and they can be understood as bright SPs due to their dipole-active character. ${ }^{5,7}$ Bright SPs can be further tailored by controlling the size and morphology of noble metal nanoparticles (NPs) with a characteristic dimension ranging from tens to a few hundreds of nanometers. ${ }^{8}$ Indeed, SP bands spanning the vis-NIR spectral regime have been reported in nanoprisms, ${ }^{9}$ nanorings, ${ }^{10}$

* To whom correspondence may be addressed: chumingwen@ntu.edu.tw and viktor@io.cfmac.csic.es.

Center for Condensed Matter Sciences, National Taiwan University.

* Instituto de Óptica-CSIC and Unidad Asociada CSIC-Universidade de Vigo.

${ }^{\S}$ Department of Physics, National Taiwan University, and Institute of Atomic and Molecular Sciences, Academia Sinica.

" Department of Chemistry, Tamkang University.

${ }^{\perp}$ Department of Chemistry, National Taiwan University. nanostars, ${ }^{11}$ single and coupled nanorods, ${ }^{5,12}$ and single and arrayed nanospheres. ${ }^{6,13-17}$ Controlling SP mode symmetries and frequencies through NP morphology $y^{5,6,9-17}$ finds exciting applications in fields as diverse as light guiding beyond the subwavelength limit ${ }^{18,19}$ and optical sensing (e.g., surfaceenhanced Raman scattering, ${ }^{20}$ SERS).

The development of these and other novel applications relies strongly on detailed knowledge of the near fields associated with SPs. ${ }^{6,8,11}$ The unambiguous determination of these near fields requires nanometer-scale resolution, ${ }^{9}$ which is, however, not achievable by conventional optical techniques such as scanning near-field optical microscopy (SNOM) that can hardly reach a spatial resolution below 50 nm. ${ }^{9,19}$ In contrast, electron-energy-loss spectroscopy (EELS) performed in a scanning transmission electron microscope (STEM) routinely provides nanometer spatial resolution by focusing a subnanometer electron beam on NPs of interest and analyzing the energy losses of the electrons to the NPs (lost to SP excitations in our case). ${ }^{9,21}$ 
The principle of STEM-EELS probing is to first position the electron beam in an electron microscope close to the NP surface or penetrating through the NP. The electromagnetic field of the incident electrons can then couple to the SP near fields of the NPs, thus polarizing them and giving rise to SP excitations, which result in electron energy losses. ${ }^{22-25} \mathrm{An}$ electron beam can, therefore, excite bright SPs by coupling primarily to their evanescent-field components close to the NP surface. This yields detailed information on the spatial distribution of SPs. In contrast, external light can couple to bright plasmons, but it does not provide the corresponding spatial information.

In addition to that, Maxwell's equations predict the existence of dipole-inactive SPs in metal NPs. The quadrupole and higher-order multiples in nanospheres with up to a few tens of nanometers in size are good examples of SPs that cannot be optically resolved and couple only marginally to light: they are dark SPs. ${ }^{7}$ However, an electron beam impinging the NPs can couple to these dark SP modes. ${ }^{26}$ STEM-EELS excitation of dark SPs is thus attainable on the same footing as that of bright SPs, and this is precisely what we report in this Letter.

Previous works have used STEM-EELS to probe SPs in noble metal NPs, ${ }^{9,21}$ both spectrally and spatially, while no details were presented on the bright or dark nature of the probed SPs. However, both a detailed analysis of the SPexcitation characteristics and their underlying implications for the corresponding optical excitations are of critical significance considering that SP probing by means of STEMEELS may lead to results that are new from the mainstream perspective within the field of plasmonics. ${ }^{18,19}$ The goal of our work is thus to systematically address the nature and spatial distribution of SP modes in noble metal NPs with different sizes and shapes, as well as understanding the coupling of fast electrons to localized SPs through joint experimental and theoretical STEM-EELS efforts.

Here, we report specifically on spectral and spatial probing of bright and dark SP modes in Au NPs (single nanorods, coupled nanorod dimers, single nanospheres, and touchingnanosphere dimers), ranging from $1.55 \mathrm{eV}(800 \mathrm{~nm})$ to 2.48 $\mathrm{eV}(500 \mathrm{~nm})$ in energy (i.e., in the vis-NIR spectral region). We use STEM-EELS with an improved energy resolution assisted by an electron monochromator. Our experimental results are in excellent agreement with STEM-EELS calculations performed by solving Maxwell's equations using Mie theory for an electron near a single sphere ${ }^{23}$ and the boundary element method (BEM $)^{24}$ for the other geometries. The spatial distributions of SP modes on individual and coupled NPs are also discussed. Both our measured and calculated SP maps obtained by STEM-EELS mimic quite closely the near-field distributions calculated for external illumination in the case of bright SP modes. Furthermore, the STEMEELS probing technique allows us to address the dark SP modes that are not resolvable by optical techniques.

We have performed STEM-EELS studies of Au NPs using a field-emission STEM, FEI Tecnai F20, operated at 200 $\mathrm{kV}$ and equipped with a Wien-filter electron monochromator.
The monochromator improves the energy resolution (defined by the line width of the zero-loss peak, ZLP) from $\sim 0.57$ to $\sim 0.22 \mathrm{eV}$ and significantly reduces the ZLP tail intensity, ${ }^{27}$ accompanied by a beam broadening of $\sim 0.2-2 \mathrm{~nm}$, due to the limited focusing power of the electromagnetic lens set. All SP spectral features shown in this work cannot be observed without the electron monochromator, because they are otherwise masked by the ZLP tail. The synthesis of the Au NPs was reported in a separate work. ${ }^{28}$ The NPs were deposited on $\sim 20 \mathrm{~nm}$ thick amorphous lacey carbon films on a $\mathrm{Cu}$ grid with repeated washings and plasma cleaning to eliminate organic contamination prior to the STEM-EELS analysis. The amorphous carbon films are free from noticeable STEM-EELS spectral excitations below $5 \mathrm{eV},{ }^{25}$ and therefore, they are optically clean to study the NPs in this spectral regime of interest. All nanomaterials investigated in this work are isolated and placed in vacuum with excellent surface cleanness (e.g., see the TEM image in the bottomleft inset of Figure 1c). These specimen features are propitious for studying the intrinsic SP excitations of the NPs. ${ }^{25}$

Figure 1a shows the calculated optical extinction spectra of an individual Au nanorod with diameter and length of 27 and $85 \mathrm{~nm}$, respectively. A nanorod of such dimensions was experimentally investigated by STEM-EELS (Figure 1c) and is shown in the inset of Figure 1c (bottom left). In Figure 1a, the interaction between the NP and incident light with electric field $E_{\text {inc }}$ polarized along (red curve) and perpendicular to (blue curve) the NP long axis produces strong light excitation assisted by two respective SP modes (A and B; inset, Figure 1a), both of which are dipole-driven bright SPs. Mode A is a longitudinal mode excited by parallel polarization and involving an induced dipole dominating along the rod axis, whereas mode $\mathrm{B}$ couples to the transversal polarization with an induced dipole perpendicular to that axis. ${ }^{5,29}$ The calculated electric near-field distributions of both nanorod bright SP modes are shown in Figure 1a (inset, top right). The red shift observed in mode $\mathrm{A}$ is well understood as originating from retardation involving the length of the nanorod. ${ }^{8}$

Panels $\mathrm{b}$ and $\mathrm{c}$ of Figure 1 show calculated and experimental STEM-EELS spectra for an incident electron beam at locations I and II relative to the nanorod, in grazing incidence with respect to the NP surface. The dominant longitudinal part of the wave field of the incident electrons is pointing out of the paper plane in contrast to the in-plane transverse wave field of light (Figure 1a). But in fact, probing the nanorod with STEM-EELS also leads to the same SP excitations (1.76 and $2.34 \mathrm{eV}$, respectively; Figure 1c) induced by optical illumination (Figure 1a). The measured spectral positions of the SP modes in Figure 1c are in agreement with those obtained from the STEM-EELS (Figure 1b) and light extinction (Figure 1a) calculations. Obviously, the electron beam can excite bright SP modes as optical illumination does. By charge symmetry, the electron at position I would exclusively excite the longitudinal mode A, whereas the electron at position II only excites mode B. However, the electron at position I can couple to higher- 


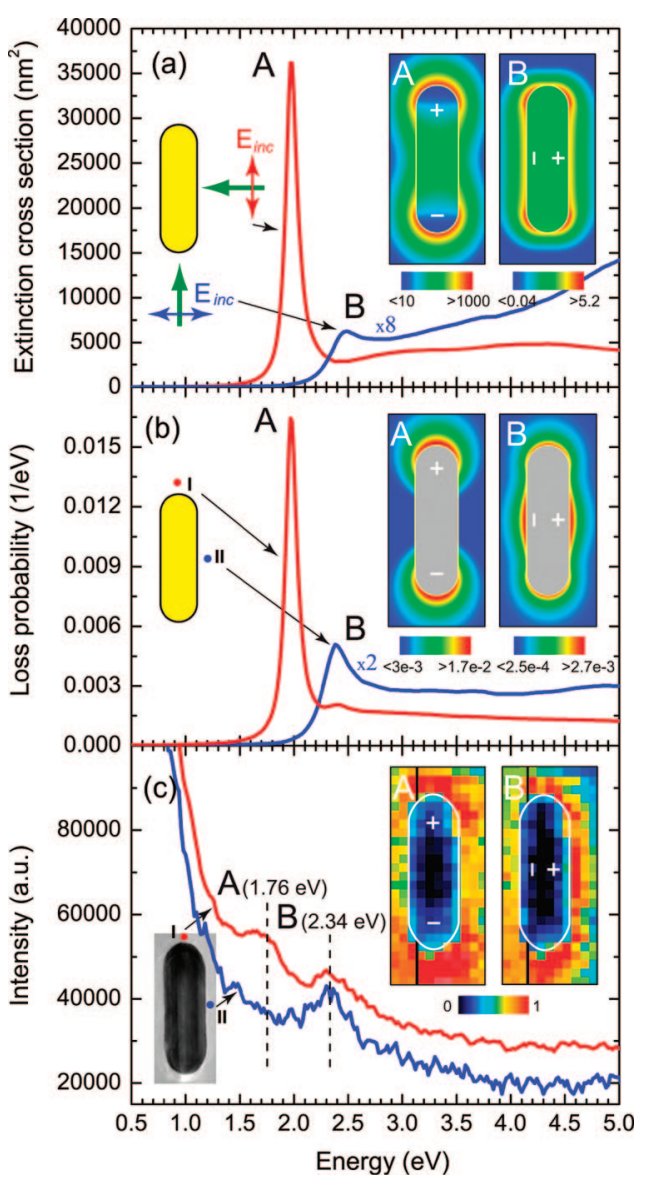

Figure 1. Comparison of light excitation vs electron excitation of nanorod longitudinal and transversal SP modes. (a) Calculated extinction cross section for an individual Au nanorod (diameter/ length, 27/85 nm; see also TEM image in the bottom-left inset in (c)). The incident-light electric field is either parallel (red curve) or perpendicular (blue curve) to the rod axis, leading to excitation of bright SP modes A and B, respectively. Top-right inset: calculated near fields of modes A and B. In the inset, the positive and negative signs denote the induced charge oscillation patterns of the modes. (b) Calculated STEM-EELS loss probability for locations I (red curve) and II (blue curve) of the electron beam relative to the nanorod under grazing incidence. The loss probability is given per incoming electron and per electronvolt for a given lost energy. Top-right inset: calculated STEM-EELS excitation-intensity maps with the gray area representing the NP. (c) Experimental counterpart of (b). The STEM-EELS spectra are normalized to the ZLP and then displaced vertically to improve readability. Top-right inset: measured STEM-EELS excitation-intensity maps for modes $\mathrm{A}$ and $\mathrm{B}$ with the solid gray lines indicating the amorphous carbonfilm boundary, to the right of which there is vacuum. Image contrast near the carbon-film side is perturbed by the film, and the mesh dimension is $4 \times 4 \mathrm{~nm}$. Color scale bar represents the linearly normalized image intensity.

order transversal modes that involve more oscillations of the induced charges along the surface and are energetically closer to the classical surface-plasmon energy $(\sim 2.4 \mathrm{eV}) .{ }^{25}$ Due to symmetry consideration, transversal modes should not be excited by the electron at position I, but it is possible that slight asymmetries can result in effective coupling, ${ }^{25}$ which explains the piling up of loss intensity near the energy of mode B even with the beam aimed at position I (Figure 1c).

The extraordinary nanometer resolution of the electron beam is clearly exemplified in the measured STEM-EELS excitation-intensity maps (see top right inset in Figure 1c; mesh dimension, $4 \times 4 \mathrm{~nm}$ ), obtained by rastering the electron beam on the nanorod. ${ }^{9,21}$ These maps nicely mimic the near-field distributions of the bright SP modes and are in excellent agreement with the near fields calculated for light illumination (see top-right inset in Figure 1a). STEM-EELS probing is thus capable of resolving NP SPs, unlike the best optical techniques that have a resolution of only tens of nanometers. ${ }^{9,19}$

In Figure 2a, we show the optical calculated extinction spectra for the coupled nanorod dimer, each of which has a size similar to that of the single nanorod of Figure 1. Moreover, each rod is inclined by an angle $\alpha=18^{\circ}$ with respect to the long dimer axis, as in the experiment that we discuss below (see the TEM image, inset of Figure 2c). The two nanorods are separated by a gap distance of $\sim 8 \mathrm{~nm}$, as shown in the TEM image. SP near-field coupling across the gap induces three SP modes (C, D, and E in Figure 2a), not just two like in individual nanorods (A and B in Figure 1a). The excitation energy of mode $\mathrm{E}$ (Figure 2a) is comparable to that of mode B for the single nanorod (Figure 1a), with both of them lying near $2.4 \mathrm{eV}$, whereas the energies of modes $\mathrm{C}$ and $\mathrm{D}$ are visibly red-shifted (mode $\mathrm{C}$ ) and blueshifted (mode D) with respect to mode A.

Mode C is a bright longitudinal coupled SP mode, which can be excited with external light polarized along the dimer axis (red curve, Figure 2a) and is characterized by a charge oscillation that is antisymmetric with respect to the gap (topright inset). The larger distances involved in the oscillation of mode $\mathrm{C}$ along the long dimer axis are the reason of its marked red shift with respect to the bright longitudinal SP mode in the single nanorod (mode A in Figure 1a). For transversally polarized incident light relative to the long dimer axis (solid and dashed blue curves in Figure 2a), one finds interesting results: modes $\mathrm{C}, \mathrm{D}$, and $\mathrm{E}$ are simultaneously observed for $\alpha=18^{\circ}$, but only mode $\mathrm{E}$ is excited in the artificially set $\alpha=0^{\circ}$. The antisymmetric charge pattern in the direction across the rods in mode $\mathrm{E}$ (see the top-right inset in Figure 2a) makes it optically bright (it has a net dipole) and rather independent of $\alpha$ for small angles, as shown in Figure 2a (solid and dashed blue curves). Similarly, mode C (a longitudinal mode of the dimer) possesses a net induced dipole and is thus optically bright as well. However, mode D does not have a dipole for $\alpha=$ $0^{\circ}$, since it is a typical quadrupolar mode in that case, thus becoming a dark mode. Slightly tilting the rods produces a net transversal dipole for this mode, so that it shows up under transversal illumination conditions (solid blue curve, Figure 2a).

Positioning the electron beam at location I of the nanorod dimer (grazing incidence), the coupling between the incident electron evanescent fields and the SP near fields gives rise to the STEM-EELS excitations of the two bright coupled SPs (modes $\mathrm{C}$ and $\mathrm{E}$ ) and the dark coupled mode $\mathrm{D}$, as shown in Figure 2, panels b and c. Further positioning the electron beam at the dimer-gap center (location II; see solid blue curves in Figure 2, panels $\mathrm{b}$ and $\mathrm{c}$ ), mode $\mathrm{C}$ becomes symmetry forbidden for the electron, but mode D is sym- 


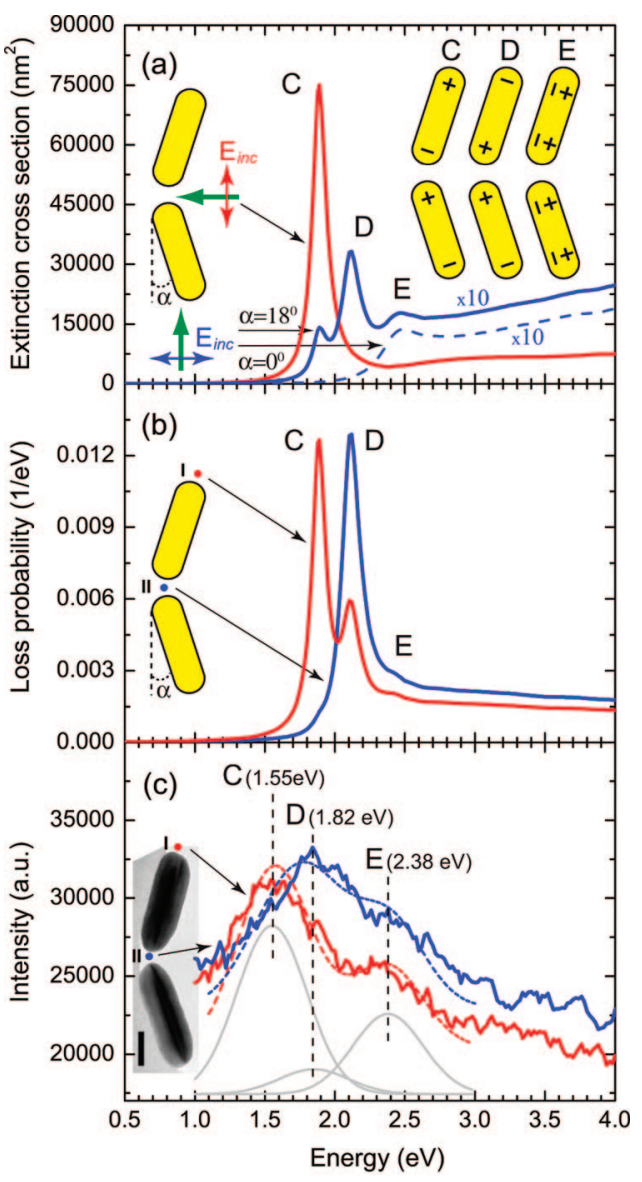

(d)

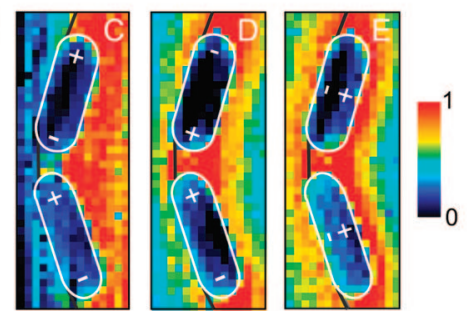

Figure 2. Electron excitation of the dark SP mode in a coupled nanorod dimer. (a) Calculated extinction cross section for a coupled $\mathrm{Au}$ nanorod dimer (the diameter/length of the upper and lower nanorods is $27 / 76$ and $27 / 81 \mathrm{~nm}$, respectively). Each individual nanorod is inclined by an angle $\alpha$ relative to the long dimer axis, and the corresponding experimental TEM image is shown in the inset of (c) (scale bar equal to $25 \mathrm{~nm}$ ). The red (blue) curve shows the scattering cross section for incident light polarization parallel (perpendicular) to the dimer axis. Solid and dashed blue curves are obtained with $\alpha=$ 18 and $0^{\circ}$, respectively. Top-right inset: schematic charge oscillation patterns of modes $\mathrm{C}, \mathrm{D}$, and $\mathrm{E}$. Note that mode $\mathrm{D}$ is a dark longitudinal coupled SP. (b) Calculated STEM-EELS loss probability for locations I (solid red curve) and II (solid blue curve) with $\alpha=18^{\circ}$. The loss probability is given per incoming electron and per electronvolt for a given lost energy. (c) Experimental counterpart of (b). The STEMEELS spectra are first normalized to the ZLP that was removed afterward, and a vertical shift has been introduced to improve readability. Gaussian fitting (gray and dashed blue and red curves) has been applied to derive the spectral position of each SP mode. (d) STEM-EELS excitation-intensity maps for modes C, D, and E with the solid gray line indicating the amorphous carbon-film boundary, to the right of which there is vacuum. Image contrast near the carbonfilm side is perturbed by the film, and the mesh dimension is $4 \times 4$ $\mathrm{nm}$. Color scale bar, the linearly normalized image intensity. The excitation of mode $\mathrm{D}$ in (b) has similar strength for $\alpha=0$, in which case mode D is a dark one, only unveiled by STEM-EELS. metry allowed, thus efficiently excited. At location II, mode $\mathrm{E}$ is weakly coupling to the electron. The dark mode D can be prominently excited with the electron passing by point II when the tilt angle $\alpha$ is $0^{\circ}$ (the corresponding calculated STEM-EELS spectrum nearly identical to the solid blue curve for $\alpha=18^{\circ}$ in Figure $2 b$, thus not shown), in sharp contrast to the light-excitation result $\left(\alpha=0^{\circ}\right.$, Figure 2a). These STEM-EELS results firmly indicate that we have established the observation of not only bright but also dark coupled SP modes. The STEM-EELS excitation-intensity maps of these modes are shown in Figure 2d, and obviously, this type of analysis can be performed on both bright and dark modes, in particular, the dark mode D for a collinear dimer $\left(\alpha=0^{\circ}\right)$.

Figure 3 shows a touching nanosphere dimer (see the experimental TEM image in bottom-left panel of Figure 3c) that can be understood as the conductive coupling of two individual NPs. ${ }^{6}$ The spherical shape of each constituent NP was confirmed by sample tilting in the microscope (diameter of the top/bottom NPs, $\sim 39 / \sim 42 \mathrm{~nm}$; bottom-left panel of Figure $3 \mathrm{c}$ ), and the conductive overlap leads to a longer axis length $(\sim 80 \mathrm{~nm})$ and a shorter radial one for the dimer. Upon external illumination with the electric field polarized along the dimer axis, the induced charge oscillation thus involves a longitudinal coupled SP (mode F; Figure 3a) along the long axis and a transversal coupled SP (mode G; Figure 3a) perpendicular to that axis. The former has visibly lower excitation energy due to retardation and shape effects. ${ }^{6,8}$ The modes $\mathrm{F}$ and $\mathrm{G}$, which bear a dominant dipolar character (this is schematized by the major positive and negative signs, top-right inset of Figure 3a), are uncoupled to each other due to the cylindrical symmetry of the dimer, and they have been revealed as separate spectral features upon external illumination (Figure 3a). Compared to the SP excitation energy of a single spherical NP (mode H, Figure 3a; diameter, $\sim 40 \mathrm{~nm}$; see also the TEM image in Figure 3c, bottom-left inset), modes $F$ and $G$ in the touching dimer (Figure 3a) are red- and blue-shifted, respectively, as also theoretically indicated. ${ }^{6,8}$ The associated near-field characteristics of the bright SPs (modes F and G) calculated for optical illumination are illustrated in Figure 3a (top-right inset).

The longitudinal dimer mode $\mathrm{F}$ is efficiently excited with the electron aimed at position I (see the left inset in Figure $3 \mathrm{~b}$ and the TEM image in Figure 3c). The spectral position of the STEM-EELS spectrum (mode F, Figure 3, panels b and c) agrees well with that of the longitudinal feature in the optical spectrum (Figure 3a). Most importantly, STEMEELS again allows us collecting the SP map of mode F with a nanometer spatial resolution, exhibiting, in both theory (Figure 3b) and experiment (Figure 3c), intensity maxima near the upper and lower ends of the dimer. However, it is important to stress that not all features in the near-field map of mode F (top-right inset, Figure 3a) are sampled equally well by the electron: only those features associated to evanescent electric field components mainly polarized along the electron trajectory contribute to electron energy losses (i.e., the upper and lower ends of the dimer). ${ }^{30,31}$ The piling 


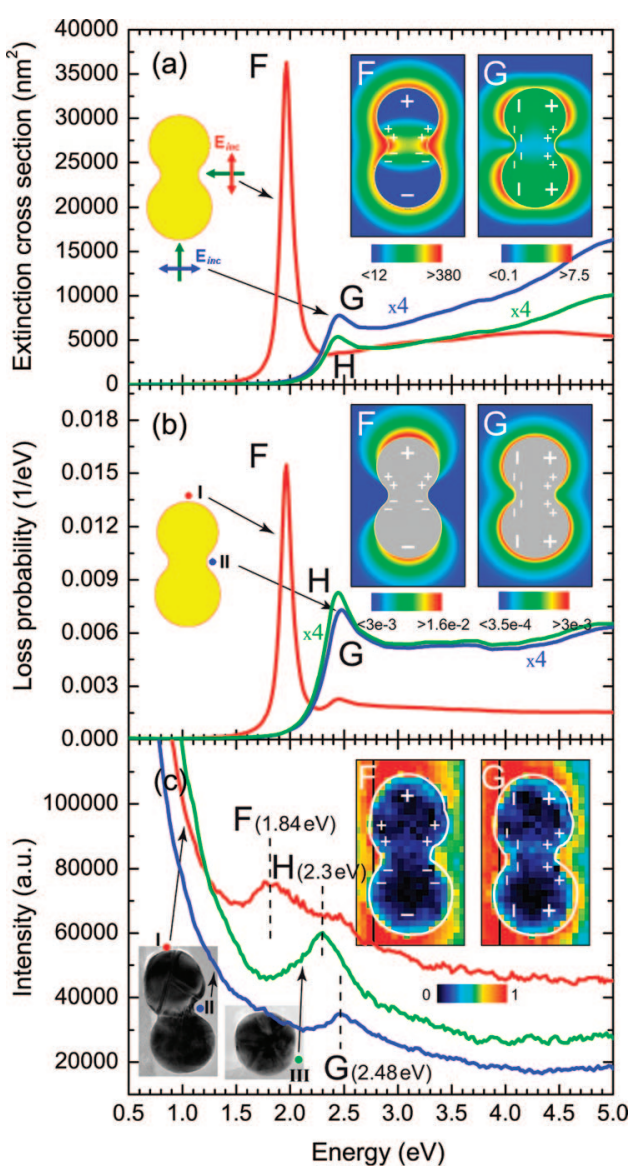

Figure 3. Electron excitation of SPs in a touching nanosphere dimer and a single nanosphere. The experimental TEM image of the dimer is shown in the bottom-left inset of (c). The diameters of the top and bottom nanospheres are $\sim 39$ and $\sim 42 \mathrm{~nm}$, respectively. The experimental TEM image of an individual nanosphere with $\sim 40$ $\mathrm{nm}$ in diameter is also shown in (c). (a) Calculated extinction cross section for a touching Au nanosphere dimer. The red (blue) curve shows the extinction cross section for incident light polarization parallel (perpendicular) to the dimer axis. The green curve corresponds to unpolarized-light scattering by a single NP. Topright inset: calculated near-field patterns of modes $\mathrm{F}$ and $\mathrm{G}$. Large positive and negative signs in the inset illustrate the induced charge associated to these modes, whereas small signs denote redistributed charge around the dimer-neck region. (b) Calculated STEM-EELS loss probability for locations I (red curve) and II (blue curve) relative to the dimer in grazing incidence. The probability is given per incoming electron and per electronvolt for a given lost energy. Topright inset: calculated STEM-EELS excitation-intensity maps for modes $\mathrm{F}$ and $\mathrm{G}$ with the gray area representing the dimer. (c) Experimental counterpart of (b). The STEM-EELS spectra are normalized to the ZLP and then vertically shifted to improve readability. Experimental STEM-EELS excitation-intensity maps for modes $\mathrm{F}$ and $\mathrm{G}$ are shown in the top-right insets, with the solid gray line indicating the amorphous carbon-film boundary, to the right of which there is vacuum. Image contrast near the carbonfilm side is perturbed by the film, and the mesh dimension is $4 \times$ $4 \mathrm{~nm}$. Color scale bar represents the linearly normalized image intensity.

up of the electric field intensity associated to the F mode near the dimer neck (see Figure 3a, top-right inset) is largely missed by the electron (see also the blue curve acquired at position II, Figure 3c), because the local field component therein is mainly perpendicular to the electron trajectory and the local charge oscillation pattern ${ }^{6}$ (see the minor positive and negative signs near the dimer neck, Figure 3, panels $b$ and c) is symmetry forbidden for the electron. ${ }^{30}$ This is an important feature in particle dimers, used for instance in ultrasensitive SERS analysis, in which the visualization of coupled bright SP distributions such as mode F here and mode $\mathrm{C}$ in Figure 2 is of primary interest. ${ }^{8}$ We anticipate that these types of SP-distribution features should be observable by tilting the sample and measuring the spectra at various angles of electron incidences. In such cases, STEMEELS should then be able to provide information on the local polarization of the SP near fields.

With the electron beam aimed at position II of panels b and $\mathrm{c}$ of Figure 3 (i.e., near the neck of the dimer), only mode $\mathrm{G}$ is excited efficiently due to symmetry considerations for the electron-dimer system. The energy of this mode $G$ is close to the main dipole mode observed in a single nanosphere (mode H, Figure 3). Overall, we observe excellent agreement between experiment and theory for the SP modes and the relative position of the SP features in the STEMEELS spectra of various individual and coupled Au nanoobjects.

In conclusion, the above results unambiguously indicate the unmatched capability of the electron beam for spectrally and spatially probing the symmetries and the spatial patterns of bright and dark SP modes in NPs with a wide variety in their geometrical constraints. Using STEM-EELS with a 2 $\mathrm{nm}$ electron beam and an improved energy resolution, we have resolved the rich SP spectral features displayed by single Au nanorods, coupled nanorod dimers, single nanospheres, and touching nanosphere dimers. These SPs show up in the vis-NIR range of $\sim 1.5-2.5 \mathrm{eV}$ (Figures 1c, 2c, and $3 \mathrm{c}$ ), and we have established their respective characters as bright (dipolar) or dark (nondipolar) modes by extensive comparisons between the experimental STEM-EELS results and those obtained by theoretical STEM-EELS and optical excitation calculations. It is important to note that dark SPs cannot be probed through far-field light scattering, while they are visible using electron beams. Moreover, the spatially resolving character of the electron beam enables us to investigate the spatial distribution of each SP mode on isolated and coupled NPs. In summary, we have proved the existence of dark SP modes and we have spatially resolved the corresponding near-field distributions using STEM-EELS, altogether providing insight beyond what can be reached by optical techniques and thus opening new possibilities for incorporating these dark modes into the body of knowledge currently available in the field of plasmonics.

Acknowledgment. This work was supported by the National Taiwan University Excellence Project, the National Science Council of Taiwan, the Spanish Ministry of Science and Innovation (MAT2007-66050 and Consolider NanoLight), and the EU-FP6 (NMP4-2006-016881 "SPANS"). The authors deeply appreciate technical support from Dr. D. Tang (FEI Co.).

\section{References}

(1) Raether, H. Excitation of Plasmons and Interband Transitions by Electrons; Springer Tracts in Modern Physics (volume 88); SpringerVerlag: Berlin and Heidelberg, Germany, 1980. 
(2) Ritchie, R. H. Phys. Rev. 1957, 106, 874-881.

(3) Stern, E. A.; Ferrell, R. A. Phys. Rev. 1960, 120, 130-136.

(4) Schwerdtfeger, P. Angew. Chem., Int. Ed. 2003, 42, 1892-1895.

(5) Aizpurua, J.; Bryant, G. W.; Richter, L. J.; García de Abajo, F. J.; Kelley, B. K.; Mallouk, T. Phys. Rev. B 2005, 71, 235420.

(6) Romero, I.; Aizpurua, J.; Bryant, G. W.; Garcı'a de Abajo, F. J. Opt. Express 2006, 14, 9988-9999.

(7) Nordlander, P.; Oubre, C.; Prodan, E.; Li, K.; Stockman, M. I. Nano Lett. 2004, 4, 899-903.

(8) Myroshnychenko, V.; Rodríquez-Fernández, J.; Pastoriza-Santos, I.; Funston, A. M.; Novo, C.; Mulvaney, P.; Liz-Marzán, L. M.; García de Abajo, F. J. Chem. Soc. Rev. 2008, 37, 1792-1805.

(9) Nelayah, J.; Kociak, M.; Stéphan, O.; García de Abajo, F. J.; Tencé, M.; Henrard, L.; Taverna, D.; Pastoriza-Santos, I.; Liz-Marzán, L. M.; Colliex, C. Nat. Phys. 2007, 3, 348-353.

(10) Aizpurua, J.; Hanarp, P.; Sutherland, D. S.; Käll, M.; Bryant, G. W.; García de Abajo, F. J. Phys. Rev. Lett. 2003, 90, 057401.

(11) Hao, F.; Nehl, C. L.; Hafner, J. H.; Nordlander, P. Nano Lett. 2007, 7, 729-732.

(12) Canfield, B. K.; Husu, H.; Laukkanen, J.; Bai, B.; Kuittinen, M.; Turunen, J.; Kauranen, M. Nano Lett. 2007, 7, 1251-1255.

(13) Klar, T.; Perner, M.; Grosse, S.; von Plessen, G.; Spirkl, W.; Feldmann, J. Phys. Rev. Lett. 1998, 80, 4249-4252.

(14) Imura, K.; Okamoto, H.; Hossain, M. K.; Kitajima, M. Nano Lett. 2006, 6, 2173-2176.

(15) Atay, T.; Song, J.-H.; Nurmikko, A. V. Nano Lett. 2004, 4, $1627-$ 1631.
(16) Khan, I.; Cunningham, D.; Lazar, S.; Graham, D.; Smith, W. E.; McComb, D. W. Faraday Discuss. 2006, 132, 171-178.

(17) Danckwerts, M.; Novotny, L. Phys. Rev. Lett. 2007, 98, 026104.

(18) Barnes, W. L.; Dereux, A.; Ebbesen, T. W. Nature 2003, 424, 824830 .

(19) Ozbay, E. Science 2006, 311, 189-193.

(20) Wang, H.-H.; Liu, C.-Y.; Wu, S.-B.; Liu, N.-W.; Peng, C.-Y.; Chan, T.-H.; Hsu, C.-F.; Wang, J.-K.; Wang, Y.-L. Adv. Mater. 2006, 18, 491-495.

(21) Bosman, M.; Keast, V. J.; Watanabe, M.; Maaroof, A. I.; Cortie, M. B. Nanotechnology 2007, 18, 165505.

(22) Kociak, M.; Stéphan, O.; Henrard, L.; Charbois, V.; Rothschild, A.; Tenne, R.; Colliex, C. Phys. Rev. Lett. 2001, 87, 075501.

(23) García de Abajo, F. J. Phys. Rev. B 1999, 59, 3095-3107.

(24) García de Abajo, F. J.; Howie, A. Phys. Rev. B 2002, 65, 115418.

(25) Chu, M.-W.; Chen, C. H.; García de Abajo, F. J.; Deng, J.-P.; Mou, C.-Y. Phys. Rev. B 2008, 77, 245402.

(26) Zabala, N.; Ogando, E.; Rivacoba, A.; García de Abajo, F. J. Phys. Rev. B 2001, 64, 205410.

(27) Kimoto, K.; Kothleitner, G.; Grogger, W.; Matsui, Y.; Hofer, F. Micron 2005, 36, 185-189.

(28) Deng, J.-P.; Shih, W.-C.; Mou, C.-Y. ChemPhysChem 2005, 6, 20212025.

(29) Link, S.; Mohamed, M. B.; El-Sayed, M. A. J. Phys. Chem. B 1999, 103, 3073-3077.

(30) Batson, P. E. Ultramicroscopy 1992, 47, 133-144.

(31) García de Abajo, F. J.; Kociak, M. Phys. Rev. Lett. 2008, 100, 106804. NL803270X 